Bull. Austral. Math. Soc.

VOL. 72 (2005) [491-496]

\title{
LIPSCHITZ FUNCTIONS WITH MAXIMAL CLARKE SUBDIFFERENTIALS ARE STAUNCH
}

\author{
Jonathan M. Borwein AND Xianfu Wang
}

In a recent paper we have shown that most non-expansive Lipschitz functions (in the sense of Baire's category) have a maximal Clarke subdifferential. In the present paper, we show that in a separable Banach space the set of non-expansive Lipschitz functions with a maximal Clarke subdifferential is not only generic, but also staunch in the space of non-expansive functions.

\section{IntRoduction AND Definitions}

Lipschitz functions with maximal subdifferentials provide counter-examples in nonsmooth analysis and differentiability theory. In a recent paper [1], we showed that the set of Lipschitz functions with maximal subdifferentials is residual in the space of all non-expansive functions. The purpose of this note is to strengthen this by showing that, in a separable-setting the set of all non-expansive Lipschitz functions with maximal subdifferentials is not only of residual but also staunch, by which we mean the complement of the set is $\sigma$-porous. We now recall the appropriate notion of porosity.

Let $(Y, d)$ be a complete metric space. We denote by $B(y, r)$ the closed ball of center $y \in Y$ and radius $r>0$. A subset $E \subset Y$ is called porous in $(Y, d)$ if there exist $0<\alpha \leqslant 1$ and $r_{0}>0$ such that for each $0<r \leqslant r_{0}$ and each $y \in Y$, there exists $z \in Y$ for which

$$
B(z, \alpha r) \subset B(y, r) \backslash E .
$$

A subset of the space $Y$ is called $\sigma$-porous in $(Y, d)$ if it is a countable union of porous subets in $(Y, d)$. All $\sigma$-porous sets are of the first category. If $Y$ is a finite dimensional Euclidean space, then $\sigma$-porous sets are of Lebesgue measure 0 . The class of $\sigma$-porous sets is much smaller than the class of sets which have measure 0 and are of the first category. In fact, in each topologically complete metric space without isolated points there exists a closed nowhere dense set which is not $\sigma$-porous [6, Theorem 1].

Throughout, $X$ is a separable Banach space with norm $\|\cdot\|$, and its topological dual is denoted by $X^{*}$ with dual unit ball $B^{*}$. We use $S_{X}$ to denote the unit sphere of $X$. Let

Received 5th September, 2005

Research for the first author was supported by NSERC and the CRC programme. Research for the second author was supported by NSERC.

Copyright Clearance Centre, Inc. Serial-fee code: 0004-9727/05 \$A2.00+0.00. 
$A \subset X$ be a bounded open convex set. For a real-valued $f: A \rightarrow R$ we say that $f$ is $K$-Lipschitz on $A$ if $K>0$ and $|f(x)-f(y)| \leqslant K\|x-y\|$ for all $x, y \in A$. When $K=1$, $f$ is called nonexpansive. The Clarke derivative of $f$ at point $x$ in the direction $v$ is given by

$$
f^{\circ}(x ; v):=\limsup _{\substack{y \rightarrow x \\ t \neq 0}} \frac{f(y+t v)-f(y)}{t}
$$

while the Clarke subdifferential $\partial_{c} f$ is given by:

$$
\partial_{c} f(x):=\left\{x^{*} \in X^{*} \mid\left\langle x^{*}, v\right\rangle \leqslant f^{\circ}(x ; v) \text { for all } v \in X\right\} .
$$

Note that $f^{\circ}(x ; v)$ is upper semicontinuous as a function of $(x, v)$. Being nonempty and weak $^{*}$ compact convex valued, the multifunction $\partial_{c} f: A \rightarrow 2^{X^{*}}$ is norm-to-weak ${ }^{*}$ upper semicontinuous. Detailed properties about Clarke subdifferentials can be found in Clarke [3, Chapter 2], which is a sort of bible for nonsmooth analysts.

\section{The Main Result}

Let $C$ be a weak*-compact convex subset of $X^{*}$. Recall that the support function of $C$ is the function $\sigma_{C}: X \rightarrow R$ defined by

$$
\sigma_{C}(v):=\sup \left\{\left\langle x^{*}, v\right\rangle \mid x^{*} \in C\right\} .
$$

Clearly, $\sigma_{C}$ is sublinear, and Lipschitz with Lipschitz rate $K:=\sup \left\{\left\|x^{*}\right\|: x^{*} \in C\right\}$. Consider

$$
\mathcal{N}_{C}:=\left\{f \mid f: A \rightarrow R \text { and } f(x)-f(y) \leqslant \sigma_{C}(x-y) \text { for all } x, y \in A\right\} .
$$

Since each $f \in \mathcal{N}_{C}$ satisfies $f(x)-f(y) \leqslant K\|x-y\|$ for all $x, y \in A, \mathcal{N}_{C}$ is a special class of $K$-Lipschitz functions defined on $A$.

For $f, g \in \mathcal{N}_{C}$, set

$$
\rho(f, g):=\sup _{x \in A}|f(x)-g(x)| .
$$

One can easily verify that $\left(\mathcal{N}_{C}, \rho\right)$ is a complete metric space.

Our central result may now be stated.

Theorem 1. Assume that $X$ is a separable Banach space and let $A \subset X$ be a bounded open convex subset of $X$. In the complete metric space $\left(\mathcal{N}_{C}, \rho\right)$, there exists a subset $G$ such that $\mathcal{N}_{C} \backslash G$ is $\sigma$-porous in $\left(\mathcal{N}_{C}, \rho\right)$, and such that each $f \in G$ has $\partial_{c} f \equiv C$ on $A$.

Proof: Fix $x \in A, v \in S_{X}$ and a natural number $k$. Consider

$$
G(x, v, k):=\left\{f \in \mathcal{N}_{C} \mid \frac{f(x+t v)-f(x)}{t}-\sigma_{C}(v) \geqslant-\frac{1}{k} \text { for some } 0<t<\frac{1}{k}\right\} .
$$


We shall show that $\mathcal{N}_{C} \backslash G(x, v, k)$ is porous in $\left(\mathcal{N}_{C}, \rho\right)$.

According to (1), it suffices to find $0<\alpha \leqslant 1$ such that for each $r \in(0,1 / k)$ and each $f \in \mathcal{N}_{C}$ there exists $h_{2} \in \mathcal{N}_{C}$ for which

$$
B\left(h_{2}, \alpha r\right) \subset B(f, r) \cap G(x, v, k) .
$$

Of course, here $h_{2}$ relies on $r$, but $\alpha$ only relies on $(x, v, k)$.

To meet this goal, we define $h: X \rightarrow R$ by

$$
h(\tilde{x}):=f(x)-\frac{r}{4}+\sigma_{C}(\tilde{x}-x),
$$

and set

$$
h_{1}:=\min \{f, h\}, \quad h_{2}:=\max \left\{f-\frac{r}{2}, h_{1}\right\} .
$$

Clearly, $h_{2} \in \mathcal{N}_{C}$ and $f-r / 2 \leqslant h_{2} \leqslant f$, so that

$$
\rho\left(h_{2}, f\right) \leqslant \frac{r}{2}
$$

Set

$$
\alpha:=\frac{\min \left\{d_{X \backslash A}(x), 1\right\}}{8\left(\sigma_{C}(v)+\sigma_{C}(-v)+1\right)} \cdot \frac{1}{k}
$$

If we let.

$$
t:=\frac{\min \left\{d_{X \backslash A}(x), 1\right\}}{4\left(\sigma_{C}(v)+\sigma_{C}(-v)+1\right)} r,
$$

where $d_{X \backslash A}(x):=\inf \{\|x-y\|: y \in X \backslash A\}$, then $0<t<1 / k$ and $x+t v \in A$. Note that $d_{X \backslash A}(x)>0$ because $A$ is open and $x \in A$. Now

$$
h(x+t v)=f(x)-\frac{r}{4}+t \sigma_{C}(v) .
$$

Since

$$
f(x)-f(x+t v) \leqslant \sigma_{C}(-t v)
$$

we have

$$
f(x+t v) \geqslant f(x)-\sigma_{C}(-t v)=f(x)-t \sigma_{C}(-v) .
$$

The choice of $t$ implies

$$
t\left(\sigma_{C}(v)+\sigma_{C}(-v)\right) \leqslant \frac{r}{4}
$$

so that

$$
f(x)-\frac{r}{4}+t \sigma_{C}(v) \leqslant f(x)-t \sigma_{C}(-v) .
$$

It follows that $h(x+t v) \leqslant f(x+t v)$, and so $h_{1}(x+t v)=h(x+t v)$ by (2). On the other hand,

$$
f(x+t v)-\frac{r}{2} \leqslant f(x)-\frac{r}{4}+t \sigma_{C}(v)
$$


since $f(x+t v)-f(x) \leqslant \sigma_{C}(t v)$. Therefore, by (2),

$$
h_{2}(x+t v)=f(x)-\frac{r}{4}+t \sigma_{C}(v) \quad \text { and } h_{2}(x)=f(x)-\frac{r}{4} .
$$

This means

$$
\frac{h_{2}(x+t v)-h_{2}(x)}{t}=\sigma_{C}(v) .
$$

Assume that $g \in B\left(h_{2}, \alpha r\right)$. We shall show that $g \in G(x, v, k)$. Indeed, by (5), (4), (3),

$$
\begin{aligned}
\frac{g(x+t v)-g(x)}{t}-\sigma_{C}(v) & =\frac{\left(g-h_{2}\right)(x+t v)-\left(g-h_{2}\right)(x)}{t}+\frac{h_{2}(x+t v)-h_{2}(x)}{t}-\sigma_{C}(v) \\
& \geqslant \frac{-2 \alpha r}{t}=-2 \alpha r t^{-1}=-2 \alpha r\left[\frac{\min \left\{d_{X \backslash A}(x), 1\right\}}{4\left(\sigma_{C}(v)+\sigma_{C}(-v)+1\right)} r\right]^{-1} \\
& =-\alpha \cdot \frac{8\left(\sigma_{C}(v)+\sigma_{C}(-v)+1\right)}{\min \left\{d_{X \backslash A}(x), 1\right\}}=-\frac{1}{k} .
\end{aligned}
$$

Therefore,

$$
\left\{g \in \mathcal{N}_{C}: \rho\left(g, h_{2}\right) \leqslant \alpha r\right\} \subset G(x, v, k) .
$$

If $\rho\left(g, h_{2}\right) \leqslant \alpha r$, then

$$
\rho(g, f) \leqslant \rho\left(g, h_{2}\right)+\rho\left(h_{2}, f\right) \leqslant \alpha r+\frac{r}{2} \leqslant \frac{r}{2}+\frac{r}{2}=r .
$$

Thus

$$
\left\{g \in \mathcal{N}_{C}: \rho\left(g, h_{2}\right) \leqslant \alpha r\right\} \subset\left\{g \in \mathcal{N}_{C}: \rho(g, f) \leqslant r\right\} .
$$

When combined with (6), this inclusion implies that

$$
\mathcal{N}_{C} \backslash G(x, v, k) \text { is indeed porous in }\left(\mathcal{N}_{C}, \rho\right) \text {. }
$$

Now let $\left\{x_{n}: n \geqslant 1\right\}$ be norm dense in $A,\left\{v_{m}: m \geqslant 1\right\}$ be norm dense in $S_{X}$. Set

$$
G:=\bigcap_{n=1}^{\infty} \bigcap_{m=1}^{\infty} \bigcap_{k=1}^{\infty} G\left(x_{n}, v_{m}, k\right) .
$$

In view of (7) and that

$$
\mathcal{N}_{C} \backslash G=\bigcup_{n=1}^{\infty} \bigcup_{m=1}^{\infty} \bigcup_{k=1}^{\infty}\left(\mathcal{N}_{C} \backslash G\left(x_{n}, v_{m}, k\right)\right)
$$

the set $\mathcal{N}_{C} \backslash G$ must be $\sigma$-porous in $\left(\mathcal{N}_{C}, \rho\right)$. If $f \in G$, then for each $x_{n}, v_{m}, k$, we have $f \in G\left(x_{n}, v_{m}, k\right)$; that is,

$$
\frac{f\left(x_{n}+t_{n, m, k} v_{m}\right)-f\left(x_{n}\right)}{t_{n, m, k}}-\sigma_{C}\left(v_{m}\right) \geqslant-\frac{1}{k},
$$


for some $0<t_{n, m, k}<1 / k$. When $k \rightarrow \infty$, from the definition of $f^{\circ}$ it follows that

$$
f^{\circ}\left(x_{n} ; v_{m}\right) \geqslant \limsup _{t \downarrow 0} \frac{f\left(x_{n}+t v_{m}\right)-f\left(x_{n}\right)}{t} \geqslant \sigma_{C}\left(v_{m}\right)
$$

and consequently,

$$
f^{\circ}\left(x_{n} ; v_{m}\right) \geqslant \sigma_{C}\left(v_{m}\right) \text { for all } n, m \geqslant 1 .
$$

Since $\left\{x_{n}: n \geqslant 1\right\}$ is dense in $A$ and $\left\{v_{m}: m \geqslant 1\right\}$ is dense in $S_{X}$, for every $x \in A$ and $v \in S_{X}$, we may find subsequences (without relabelling) $\left(x_{n}\right)$ and $\left(v_{m}\right)$ such that $x_{n} \rightarrow x$ and $v_{m} \rightarrow v$. By the upper semicontinuity of $f^{\circ}$ and continuity of $\sigma_{C}$, from (8) we get

$$
f^{\circ}(x ; v) \geqslant \sigma_{C}(v)
$$

Since $f \in \mathcal{N}_{C}$, for every $y \in A, t>0$,

$$
f(y+t v)-f(y) \leqslant \sigma_{C}(t v) .
$$

Dividing both sides by $t$, and taking the limsup as $y \rightarrow x$ and $t \downarrow 0$ produces

$$
f^{\circ}(x ; v) \leqslant \sigma_{C}(v)
$$

Together with (9), we obtain

$$
f^{\circ}(x ; v)=\sigma_{C}(v) \text { for } x \in A, v \in S_{X} .
$$

Dually, $\partial_{c} f(x)=C$ for every $x \in A$, and the proof of the theorem is complete.

Observe that

$$
\mathcal{N}_{B^{*}}:=\{f \mid f: A \rightarrow R \text { is nonexpansive with respect to }\|\cdot\|\} .
$$

Theorem 1 gives:

COROLlaRY 1. In the space of nonexpansive functions, $\left(\mathcal{N}_{B^{*}}, \rho\right)$, the set

$$
\left\{f \in \mathcal{N}_{B^{*}} \mid \partial_{c} f \equiv B^{*} \text { on } A\right\}
$$

has a $\sigma$-porous complement in $\left(\mathcal{N}_{B^{*}}, \rho\right)$.

It is well-known that every locally Lipschitz function $f$ on an open subset $A$ of a separable Banach space $X$ is Gâteaux differentiable everywhere on $A$ except for possibly a Haar-null subset. We need a result due to Giles and Sciffer [4].

LEMMa 1. Let $f: A \rightarrow R$ be a locally Lipschitz function on an open subset $A$ of a separable Banach space $X$. Then the set

$$
\left\{x \in A \mid f^{+}(x ; v)=f^{\circ}(x ; v) \text { for all } v \in X\right\}
$$

is residual in $A$. Here

$$
f^{+}(x ; v):=\underset{t \downarrow 0}{\limsup } \frac{f(x+t v)-f(x)}{t}
$$


Combining Corollary 1 with Lemma 1 gives the following result.

COROLLARY 2. In the space of nonexpansive functions, $\left(\mathcal{N}_{B^{*}}, \rho\right)$, the set

$\left\{f \in \mathcal{N}_{B} \cdot \mid f\right.$ is Gâteaux differentiable at most on a first category subset of $\left.A\right\}$, has a $\sigma$-porous complement in $\left(\mathcal{N}_{B^{*}}, \rho\right)$.

Proof: Let $f \in \mathcal{N}_{B}$. such that $\partial_{c} f \equiv B^{*}$ on $A$. Consider the set

$$
S_{f}:=\left\{x \in A \mid f^{+}(x ; v)=f^{\circ}(x ; v) \text { for all } v \in X\right\} .
$$

By Lemma $1, S_{f}$ is a residual set in $A$. If $f$ is Gâteaux differentiable at $x$, then $f^{+}(x ; v)$ $=\langle\nabla f(x), v\rangle$ for every $v \in X$, and so $x \notin S_{f}$ since $\partial_{c} f(x)=B^{*}$. Therefore, such an $f$ is at most Gâteaux differentiable on $A \backslash S_{f}$, which is a first category subset in $A$. Since the set

$$
\left\{f \in \mathcal{N}_{B^{*}} \mid \partial_{c} f \equiv B^{*} \text { on } A\right\},
$$

has a $\sigma$-porous complement in $\left(\mathcal{N}_{\left.B^{*}, \rho\right)}\right.$ by Corollary 1 , the result is proved.

Finally, for various generic aspects of Lipschitz functions with maximal Clarke subdifferentials on general Banach spaces, we refer readers to [2].

\section{REFERENCES}

[1] J.M. Borwein and X. Wang, 'Lipschitz functions with maximal subdifferentials are generic', Proc. Amer. Math. Soc. 128 (2000), 3221-3229.

[2] J.M. Borwein, W.B. Moors and X. Wang, 'Generalized subdifferentials: a Baire categorical approach', Trans. Amer. Math. Soc. 353 (2001), 3875-3893.

[3] F.H. Clarke, Optimization and nonsmooth analysis (Wiley Interscience, New York, 1983).

[4] J.R. Giles and S. Sciffer, 'Locally Lipschitz functions are generically pseudo-regular on separable Banach spaces', Bull. Austral. Math. Soc. 47 (1993), 205-212.

[5] S. Reich, A.J. Zaslavski, The set of noncontractive mappings is $\sigma$-porous in the space of all non-expansive mappings, C. R. Acad. Sci. Paris 333 (2001), 539-544.

[6] L. Zajicek, 'Small non- $\sigma$-porous sets in topologically complete metric spaces', Colloq. Math. 77 (1998), 293-304.

Faculty of Computer Science

Dalhousie University

6050 University A venue

Halifax, NS

Canada, B3H 1W5

e-mail: jborwein@cs.dal.ca
Department of Mathematics and Statistics UBC Okanagan

3333 University Way

Kelowna, BC.

Canada V1V 1V7

e-mail: Shawn.Wang@ubc.ca 